\title{
Chapter
}

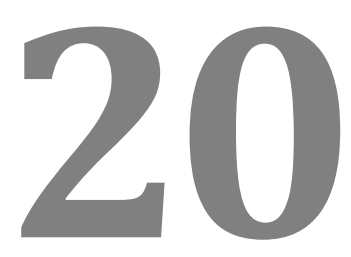

\section{COPPER SULFIDE NANOPARTICLES: FROM SYNTHESIS TO BIOMEDICAL APPLICATIONS}

Yuanyuan Qiu1,2, Lei Lu' ${ }^{1,2}$, Dehui Hu ${ }^{1,2}$, and Zeyu Xiao ${ }^{1,2,3^{*}}$

${ }^{1}$ Department of Pharmacology, Institute of Medical Sciences, Shanghai Jiao Tong University School of Medicine, 280 South Chongqing Road, Shanghai, 200025, PR China

${ }_{2}^{2}$ Translational Medicine Collaborative Innovation Center, Shanghai Jiao Tong University School of Medicine, 280 South Chongqing Road, Shanghai, 200025, PR China

${ }^{3}$ Collaborative Innovation Center of Systems Biomedicine, Shanghai Jiao Tong University School of Medicine, 280 South Chongqing Road, Shanghai, 200025, PR China 


\section{Contents}

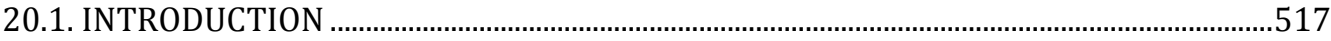

20.2 SYNTHESIS OF CuS NPs .......................................................................................................517

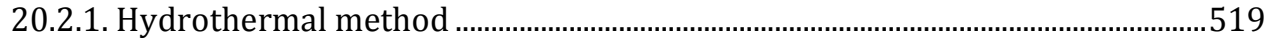

20.2.2. Microwave irradiation method .......................................................................519

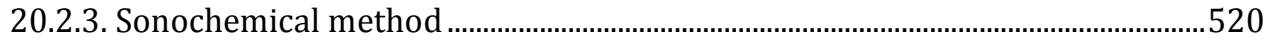

20.2.4. Other preparation methods .................................................................................521

20.3. BIOMEDICAL APPLICATIONS OF CuS NPs ……………………………………………....521

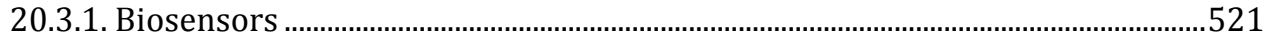

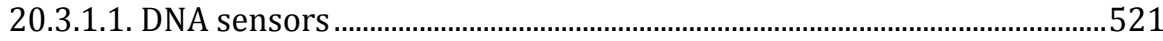

20.3.1.2. Glucose sensors ............................................................................................523

20.3.2. Molecule imaging....................................................................................................523

20.3.2.1. PAT imaging .............................................................................................524

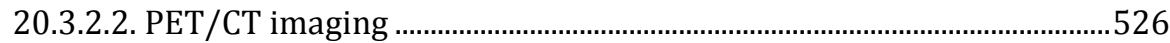

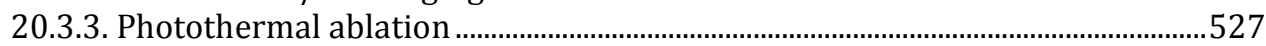

20.3.3.1. Photothermal therapy …………………………………………………....528

20.3.3.2. Transdermal delivery ……………………………………………………...529

20.3.4. Theranostics ...................................................................................................................5

20.4 SUMMARY ..........................................................................................................................531

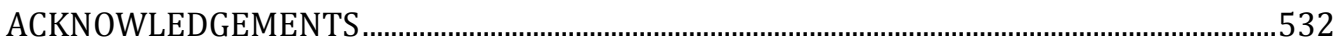

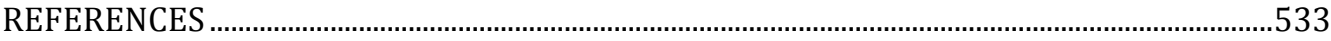




\subsection{INTRODUCTION}

The dream of developing nanoscience and nanotechnology started in 1959, when Richard Feynman presented his vision of manipulating and controlling things on a small scale at the annual meeting of American Physical Society. In 1968, molecular beam epitaxy was discovered in the Bell Laboratories, followed by the invention of scanning tunneling microscope, eventually contributing to the sophistication in the fields of nanoscience and nanotechnology [1].

As an important branch of the nanoscience and nanotechnology field, the development of nanomaterials for biomedical applications has received great attention in the past thirty years. Many nanomaterial-related products, such as textiles and cosmetics, have entered the market at an increased pace. Design and synthesis of multifunctional nanomaterials that combine two or more functions have provided potential applications in the diagnostic and treatment of cancer and a myriad of other diseases. Tunable physicochemical properties and their abilities to be efficiently applied in biological systems upon appropriate modification, make nanomaterials among the most promising systems for a range of biomedical applications, such as biosensing, molecular imaging, drug delivery, and therapy [2].

Copper sulfide nanoparticles (CuS NPs) are a class of semiconductor nanomaterials that demonstrate interesting characteristics for biomedical applications. Besides possessing the common features of semiconductor nanomaterials, including the variable energy structure and enhanced surface properties, CuS NPs are capable of forming various stoichiometries with unique optical and electrical properties, and thus they are explored as a potential platform for biosensing [3,4], molecular imaging [5], photothermal therapy [6], drug delivery [7], and theranostic agents [8].

In this review article, we summarize recent progress of CuS NPs in biomedical research. Firstly, we illustrate the detailed synthesis procedures of CuS NPs with distinct morphology and spatial orientation. Subsequently, we discuss the emerging role of CuS NPs for biomedical applications. Finally, we envision the major challenges and future directions of CuS NPs.

\subsection{SYNTHESIS OF CuS NPs}

Many studies reported the synthesis strategies of CuS NPs with all three dimensions, including (i) one dimensional nanorodes, nanotubes, and nanowires, (ii) two dimensional nanoplates, and (iii) three dimensional hollow/solid nanospheres, core-shell NPs, and nanocages (Figure 1). The synthesis methods may vary with the morphology, which in turn contributes to 
diversified properties and biomedical applications. One dimensional $\mathrm{CuS}$ nanostructures are widely used as biosensors, mainly due to their excellent electrochemical and catalytic properties; two dimensional CuS nanostructures are investigated for improving photothermal conversion efficiency; and three dimensional CuS nanostructures are suitable for biomedical applications, resulting from their easy-to-decoration properties and favorable physicochemical characteristics.

Characterization of CuS NPs can be performed using a wide variety of techniques, such as scanning electron microscopy (SEM), X-Ray diffraction (XRD), energy dispersive X-Ray spectroscopy (EDS), transmission electron microscopy (TEM) and high resolution TEM (HRTEM), atomic force microscopy (AFM), dynamic light scattering (DLS), Fourier transform infrared spectroscopy (FTIR), UV-visible and photoluminescence (PL) spectroscopy, etc. These techniques provide important information on the elemental, structural (e.g., size and shape), and optical properties of CuS NPs [2].

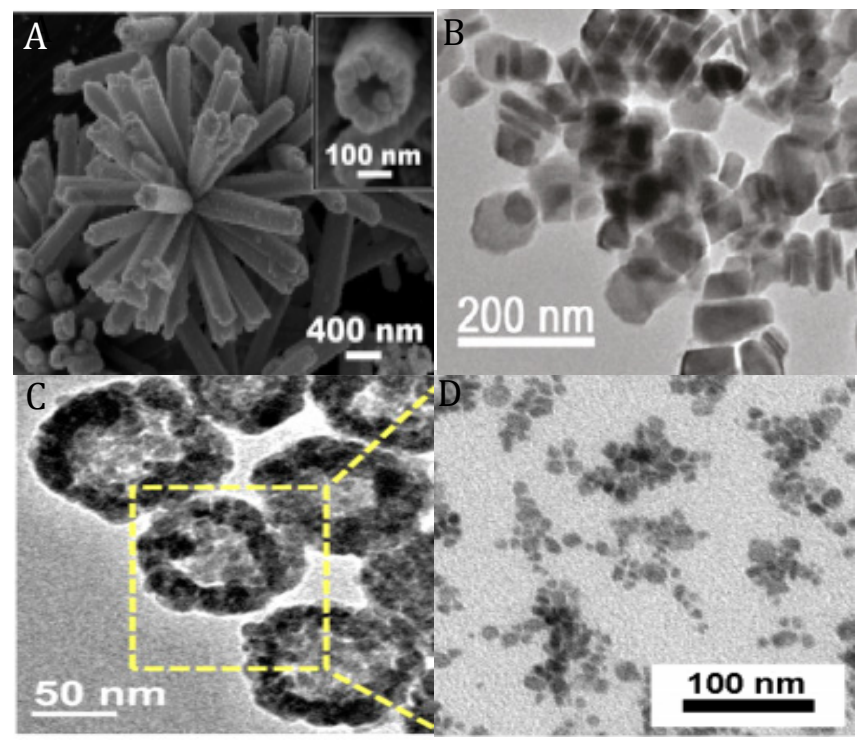

Figure 1. (A) SEM image of solution-based synthesized CuS nanotubes. This image is adapted with permission from [9] (C) (2014) American Chemical Society. (B) TEM image of $\mathrm{CuS}$ nanoplates. This image is adapted with permission from [10] (C) (2011) American Chemical Society. (C) TEM image of hollow CuS NPs. This image is adapted with permission from [11] (C) (2014) American Chemical Society. (D) TEM image of CuS nanospheres. This image is adapted with permission from [12] (C) (2012) American

Chemical Society 


\subsubsection{Hydrothermal method}

Hydrothermal method offers a cheap, green and highly scalable way to prepare inorganic nanomaterials, and it is mainly applied to synthesize metal oxide based materials. This first report of using hydrothermal method was to synthesize metal sulphide nanomaterials [13]. Subsequently, this method was applied to manufacture a wide range of binary metal sulphides (e.g., ZnS, CdS, $\mathrm{PbS}, \mathrm{CuS}, \mathrm{Fe}_{(1-\mathrm{x})} \mathrm{S}$, and $\left.\mathrm{Bi}_{2} \mathrm{~S}_{3}\right)$. By varying the reaction conditions, two mechanisms may be involved in the synthesis process: a growth dominated route which permits the formation of nanostructured sulphide materials, and a nucleation driven process which produces NPs with temperature dependent size control. Consequently, this method offers a viable route to construct a wide range of metal sulphide NPs with flexible size and shape control [14].

Hydrothermal method can be used to fabricate uniform CuS NPs, and the reaction is performed at a relatively low temperature without using complex and toxic organometallic reactants. For example, CuS nanospheres (Figure 1d) could be synthesized simply by mixing aqueous solutions of $\mathrm{CuCl}_{2}$, sodium citrate, and $\mathrm{Na}_{2} \mathrm{~S}$ together at room temperature and the following reaction at $90{ }^{\circ} \mathrm{C}$ for $15 \mathrm{~min}$ [8]. The size and shape of the nanospheres could be tuned by changing parameters such as the category of the precursors, temperature of the reaction, reaction time, and etc. In another example, hollow CuS NPs was based on the self-assembly of nanoflakes derived from $\mathrm{Cu}(\mathrm{II})$-thiourea complex into hollow nanospheres, with the size of several microns in diameter. Another study demonstrates the formation of hollow nanospheres and nanotubes from 5-10 $\mathrm{nm}$ CuS NPs at room temperature [15]. It was suggested that such hollow structures may be formed as a result of decomposition of thiourea into $\mathrm{H}_{2} \mathrm{~S}$, which further reacts with the $\mathrm{Cu}$ precursor to produce $\mathrm{CO}_{2}$. The $\mathrm{CO}_{2}$ produced forms gaseous cavities which act as heterogeneous nucleation centers under hydrothermal conditions for the aggregation of CuS nanoflakes, finally giving rise to hollow nanostructures. Two dimensional CuS nanostructures which are relatively rare and restricted mainly to nanoplates (Figure $1 \mathrm{~b}$ ) can also be prepared with hydrothermal methods. CuS nanoplates, the thickness and edge length of which are about $15 \mathrm{~nm}$ and $60 \mathrm{~nm}$, have been successfully prepared in the presence of cation surfactant cetyltrimethylammonium bromide (CTAB) by hydrothermally treating the solution of $\mathrm{CuCl}_{2} \cdot 2 \mathrm{H}_{2} \mathrm{O}$ and $\mathrm{Na}_{2} \mathrm{~S} \cdot 9 \mathrm{H}_{2} \mathrm{O}$ at $180{ }^{\circ} \mathrm{C}$ for $24 \mathrm{~h}$. The as-prepared CuS nanoplates are of hexagonal phase and are single crystal [16].

\subsubsection{Microwave irradiation method}

Microwaves are electromagnetic waves containing electric and magnetic field components. The electric field applies a force on charged particles, which was driven to migrate or rotate. The movement of charged particles further polarizes the particles. The concerted forces applied by the electric and magnetic components of microwaves rapidly change in direction, which 
creates friction and collisions of the molecules. Microwave irradiation has been developed and widely used in the fields such as molecular sieve preparation [17], radio pharmaceuticals [18], preparing inorganic complexes and oxide, organic reactions, plasma chemistry, analytical chemistry, catalysis, and recently in the preparation of nanocrystalline particles. Microwave irradiation has shown very rapid growth in its application to materials science due to its unique reaction effects such as rapid volumetric heating and the dramatic increase in reaction rates, etc. Compared with conventional methods, microwave synthesis has the advantages of short reaction time, small particle size, narrow particle size distribution and high purity.

For the synthesis of CuS NPs, this method mostly uses microwave irradiation $(\sim 180 \mathrm{~W})$ in aqueous medium or ethylene glycol for $\sim 20$ min to carry forth the decomposition process [3]. An early report stated a route to prepare $\mathrm{CuS}$ nanorods via the microwave-induced heating in aqueous solution under ambient air. Powder X-ray diffraction pattern indicates that the product was $\mathrm{CuS}$ composed of uniform and regular rods with diameters ranging from $5-10 \mathrm{~nm}$ and lengths $30-50 \mathrm{~nm}$. The product has good crystallinity, uniform morphology, and high purity [19]. In another report, pure CuS with flower-like, hollow spherical, and tubular structures (Figure 1a) were synthesis by controlling $\mathrm{pH}$ and irradiation rate. In this method, $\mathrm{CuCl}_{2} \cdot 2 \mathrm{H}_{2} \mathrm{O}$ and $\mathrm{CH}_{3} \mathrm{CSNH}_{2}$ were dissolved in ethylene glycol, and followed by the addition of $\mathrm{NaOH}$ to form solutions with different $\mathrm{pH}$ values. Reactions proceeded in surfactant-free solutions contained in an acid digestion bomb using a microwave irradiation at different conditions [20].

\subsubsection{Sonochemical method}

Sonochemical method is a facile route operated under ambient conditions. During sonication, ultrasonic sound waves radiate through the solution causing alternating high and low pressure in the liquid media. This leads to the formation, growth, and implosive collapse of bubbles in a liquid. The collapse of bubbles with short lifetimes produces intense local heating and high pressure, thus facilitating the preparation of NPs with uniform shape and narrow size distribution. This method has become an important tool in the synthesis of various nanostructures, including particles, hollow spheres, porous spheres, rods, tubes, and wires [21].

Sonochemical methods are extensively explored to synthesize CuS NPs. For example, Kuar et al. firstly reported to synthesize CuS/PVA NPs with the size of $165-225 \mathrm{~nm}$ by sonochemical irradiation of an ethylenediamine-water solution of copper acetate and sulfur under an argon flow [22]. In another report, single crystalline CuS nanoplates with average sizes of about 20-40 nm were successfully synthesized without any surfactant by a sonochemical approach under ambient conditions. $\mathrm{Cu}(\mathrm{OH})_{2}$ nanoribbons were used as the copper source and in situ template to prepare CuS nanostructure [21]. In 
another example, CuS NP-decorated reduced graphene oxide (CuS/rGO) composites have been successfully prepared via a sonochemical method in the room temperature and mild reaction conditions. X-ray diffraction and electron microscopy observations confirm that CuS NPs of $10-25 \mathrm{~nm}$ are well distributed on the rGO nanosheets. Ultraviolet-visible spectroscopy reveals the $\mathrm{CuS} / \mathrm{rGO}$ nanocomposites show a strong and broad light absorption.

\subsubsection{Other preparation methods}

Several other methods, though less frequently reported, can be used to synthesize CuS NPs. For example, the use of a paired cell at room temperature [23], thermolytic degradation of copper thiolate precursor without solvent [24], and amylose-directed synthesis [25] can be applied to synthesize one dimensional CuS NPs. Chemical vapor reaction, single source method [26], and high-temperature precursor injection method were investigated to prepare two dimensional $\mathrm{CuS}$ nanostructures. To construct three dimensional $\mathrm{CuS}$ nanostructure, some other strategies include enzymatic treatment of dextran stabilized CuS nanosuspensions in a green synthetic method [27], the use of carboxylic acids as solvents for high nucleation rate and stabilization of nanoparticle dispersion [28], and synthesis of CuS NPs based on surfactant [29].

\subsection{BIOMEDICAL APPLICATIONS OF CuS NPS}

The usage of nanomaterials in biotechnology merges the fields of material science and biology. NPs provide a particularly useful platform, demonstrating unique properties with potentially wide-ranging applications in biomedical areas, as described below [30].

\subsubsection{Biosensors}

\subsubsection{DNA sensors}

DNA detection is highly important for the diagnosis of infectious diseases, genetic mutations, and clinical medicines [31]. DNA biosensors provide a powerful means of recognizing specific DNA sequences, and different biosensors have been reported for the detection of specific DNA sequences. Among them, electrochemical DNA biosensors are attracting great interest with regards to simplification of the analysis, miniaturization, and microelectronic integration [32]. CuS is a p-type semiconductor and has been extensively applied in lithium rechargeable batteries, solar cells, biology markers, and biosensors because it is inexpensive, nontoxic, easily produced, and readily stored and has high specific capacitances [33]. However, it is not favorable for applications in electrode materials because of its low conductivity. To solve this problem, $\mathrm{CuS}$ nanocomposite was prepared on an 
electronically conductive support to facilitate charge transfer for electrochemical biosensing applications. In one example, CuS-graphene nanocomposite was constructed as a DNA biosensor platform [34]. The nanocomposite consists of $\mathrm{CuS}$, graphene nanosheets, gold NP-conjugated ssDNA probe, and mean cell hemoglobin (MCH) (Figure 2). The embedding of $\mathrm{CuS}$ on graphene nanosheets helps to improve the solubility and dispensability of graphene nanosheets, which in turn enhances the conductivity of CuS. Upon the hybridization of target DNA strand with DNA probe to form a double-stranded structure on the biosensor surface, the electrochemical response of $\left[\mathrm{Fe}(\mathrm{CN})_{6}\right]^{3-/ 4-}$ decreased, thus exhibiting a low detection limit for the target DNA.

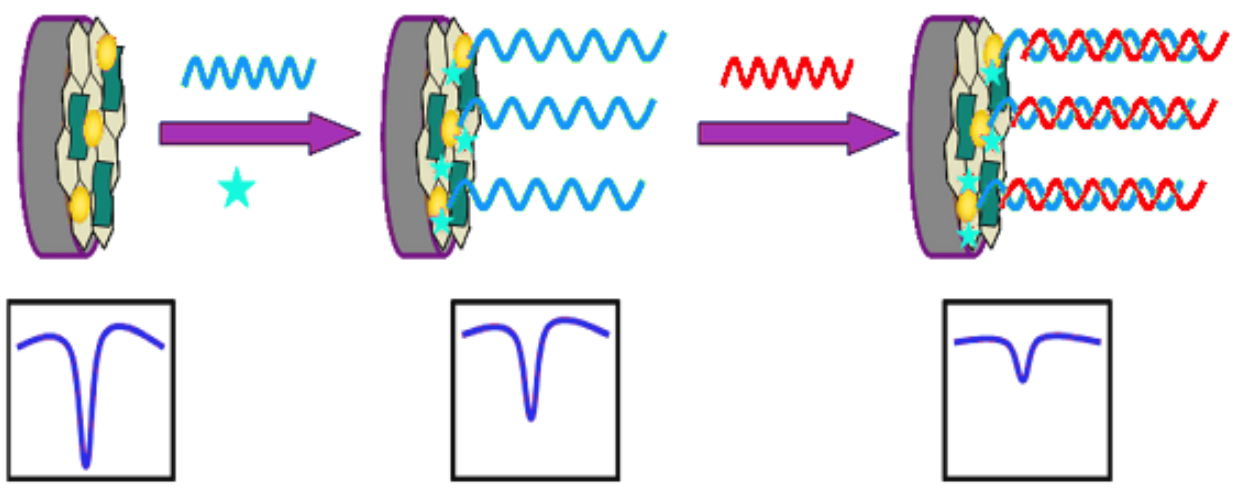

\section{* MCH MUN Probe SH-DNA MUN target cDNA}

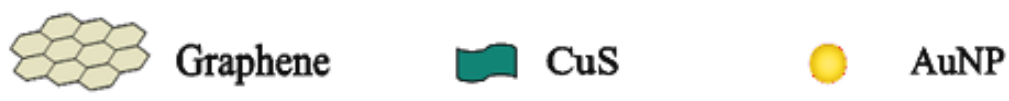

Figure 2. The electrochemical DNA biosensor consists of AuNPs, target complementary DNA, MCH and 6-mercapto-1-hexane [34]

Single-nucleotide polymorphisms (SNPs) refer to point mutations that constitute the most common genetic variation, and the single base alternations are strongly related to various medical and physiological features of human beings [35]. Therefore, accurate and sensitive analysis of SNPs will play an important role for disease diagnosis, predisposition assessment, pharmacogenetic analysis, and evolutionary studies [36]. In one study, CuS-based nanostructures were assembled to analyze SNPs. In this study, CuS NPs are modified on the surface of gold NPs, and through which are conjugated with the complementary base strand. Once the $\mathrm{CuS}$ nanostructures are 
hybridized with the mismatched bases, cupric ions will be dissolved from the CuS NPs, yielding an ultrasensitive chemiluminescence signal with the SNPs detection limit of $8.0 \times 10^{-17}$ to $1.0 \times 10^{-14} \mathrm{M}$.

\subsubsection{Glucose sensors}

Glucose detection plays an important role in monitoring diabetes. The rising demands for glucose sensor with high sensitivity, high reliability, fast response and excellent selectivity have driven enormous research efforts for decade [37]. Early efforts were focused on the development of enzymatic glucose biosensors, principled by the detection of glucose oxidase (GOD). However, GOD is paucity of longterm stability and its activity is affected by many factors (e.g., temperature, $\mathrm{pH}$ value and toxic chemicals), thus limiting the applications of this enzymatic method. Alternatively, nonenzymatic glucose sensors are recently developed. In a report, a sensitive nonenzymatic glucose sensor based on CuS nanotube (CuS NTs) was fabricated. CuS NTs made up of CuS NPs were successfully in situ synthesized on $\mathrm{Cu}$ electrode by a simple self-sacrificial template method [38]. It was a useful method to avoid the defects of the sonication and widen linear range around micromolar concentration to meet the requirement of predilution of human serum. The electrochemical property of CuS NTs modified $\mathrm{Cu}$ electrode (CuS NTs/Cu) for glucose electrooxidation was thoroughly investigated by cyclic voltammetry and amperometric methods. The CuS NTs based glucose sensor exhibited an extraordinary detection limit as low as $45 \mathrm{nM}$ and two wide linear ranges. A lower glucose concentration ranged from 0.2 to $2.5 \mathrm{mM}$ with high sensitivity of $3135 \mu \mathrm{AmM}^{-1} \mathrm{~cm}^{-2}$ and a higher glucose concentration ranged from 2.5 to $6 \mathrm{mM}$ with relative low sensitivity of $2205 \mu \mathrm{A} \mathrm{mM}-1 \mathrm{~cm}^{-2}$. Furthermore, the proposed sensor also showed high anti-interference ability and was successfully used for glucose detecting in human serum with good accuracy and satisfactory recovery.

\subsubsection{Molecule imaging}

Early diagnosis of cancer is crucial to increase the chances of survival, and NP-based molecular imaging technology is emerging as a new paradigm for cancer diagnosis [39]. Compared with conventional agents, NP-based imaging probes provide several advantages [40], including enhanced circulation halflife, the integration of "smart sensing" properties to the probes [41], and the ability to load both imaging and drug components in one vehicle for multifunctional applications [42]. Generally speaking, molecular imaging includes the use of optical techniques such as bioluminescence and fluorescence imaging [43], molecular magnetic resonance imaging (MRI) [44], magnetic resonance spectroscopy (MRS), positron emission tomography (PET) [45], single-photon emission computed tomography (SPECT) [46], and increasingly popular photoacoustic tomography (PAT) [40]. 


\subsubsection{PAT imaging}

PAT, also termed as optoacoustic tomography, is based on the measurement of ultrasonic waves induced by biological tissues absorption of short laser pulses. PAT employs nonionizing laser light to acoustically visualize biological tissues with high optical contrast and high ultrasonic resolution. Photoacoustic effects were demonstrated in turbid medium by Kruge in 1994, in biological tissues by Oraevsky et al. in 1997, and in rats by Wang et al. in 2003 [12]. Since then, this imaging modality has been widely researched and advanced toward clinical applications. The most important chromophores in the human body are oxyhemoglobin and deoxyhemoglobin in red blood cells. The hemoglobins have absorption coefficients of more than $100 \mathrm{~cm}^{-1}$ for visible light; hence, they are capable of generating strong photoacoustic signals ( $\sim 10$ bar). Therefore, PAT has been successfully applied to image vascular structures and tumor angiogenesis a few millimeters under the skin [47]. Unlike other highresolution optical imaging modalities, such as confocal microscopy, two-photon microscopy, and optical coherence tomography, PAT relies on both diffused and ballistic light and thus can be used to image deeper biological tissues. However, because of the overwhelming scattering effect of biological tissues on light, light intensity decreases exponentially with depth with a decay constant that is related to effective penetration depth. The light intensity attenuation can be minimized by choosing an excitation laser wavelength within the near-infrared region (NIR), in which biological tissues have a relatively low absorption coefficient and a low scattering coefficient. Successful translation of PAT to the clinic requires a practical laser source that efficiently penetrates biological tissues and a contrast agent whose optical absorption peaks at or near the wavelength of the laser source. Nowadays, various organic or inorganic NPs, including porphyrin shell, gold NPs, carbon nanotubes, nanographene, and CuS NPs, all with high NIR absorbance, have widely explored for in vivo PAT imaging [48].

Recent studies have demonstrated the usage of CuS NPs as excellent contrast agents for in vivo PAT imaging. In one example, CuS-based PAT imaging was applied to visualize deep tissue, including mouse brain, rat lymph nodes and chicken breast (Figure 3). CuS NPs in this study had the average diameter of $11 \pm 3 \mathrm{~nm}$, yielding an absorption peak at around $990 \mathrm{~nm}$. A $1064 \mathrm{~nm}$ -wavelength laser was chosen for irradiation, as the wavelength elicits a strong absorbance of CuS NPs while a low absorption of normal tissues, thus increasing the signal-to-noise ratio. The result shows that this CuS-based PAT imaging allows for the detection of deep tissue at a depth of $\sim 5 \mathrm{~cm}$ with an in-plane imaging resolution of $\sim 800 \mu \mathrm{m}$ and a sensitivity of $\sim 0.7 \mathrm{nmol}$ per imaging pixel [12]. In another example, CuS-based PAT imaging was used to detect the activity of matrix metalloproteinases (MMPs) in vivo [48]. MMPs are zinc-dependent endopeptides that degrade proteins in the extracellular matrix and play an important role in the development of various diseases including cancer, inflammatory, neurological and cardiovascular diseases. CuS NPs are 
conjugated with a red-light-absorbing organic dye, BHQ3, via a MMPs cleavable peptide. The obtained CuS-peptide-BHQ3 (CPQ) nano-probe exhibits strong PAT signal at the wavelengths of $680 \mathrm{~nm}$ and $930 \mathrm{~nm}$ before enzyme cleavage, resulting from the absorption of BHQ3 and CuS NPs, respectively. In the presence of MMPs expressed inside the tumor, CPQ nano-probe is recognized by the protease, releasing free BHQ3 which as a small molecule could be rapidly washed out from the tumor area, leaving bare CuS NPs which would retain inside the tumor for a much longer period of time. The PAT signal ratios recorded at two different wavelengths, $680 \mathrm{~nm} / 930 \mathrm{~nm}$, is then significantly decreased. This novel nano-probe demonstrates the first study of using PAT imaging to investigate MMP activity in the animal tumor model.
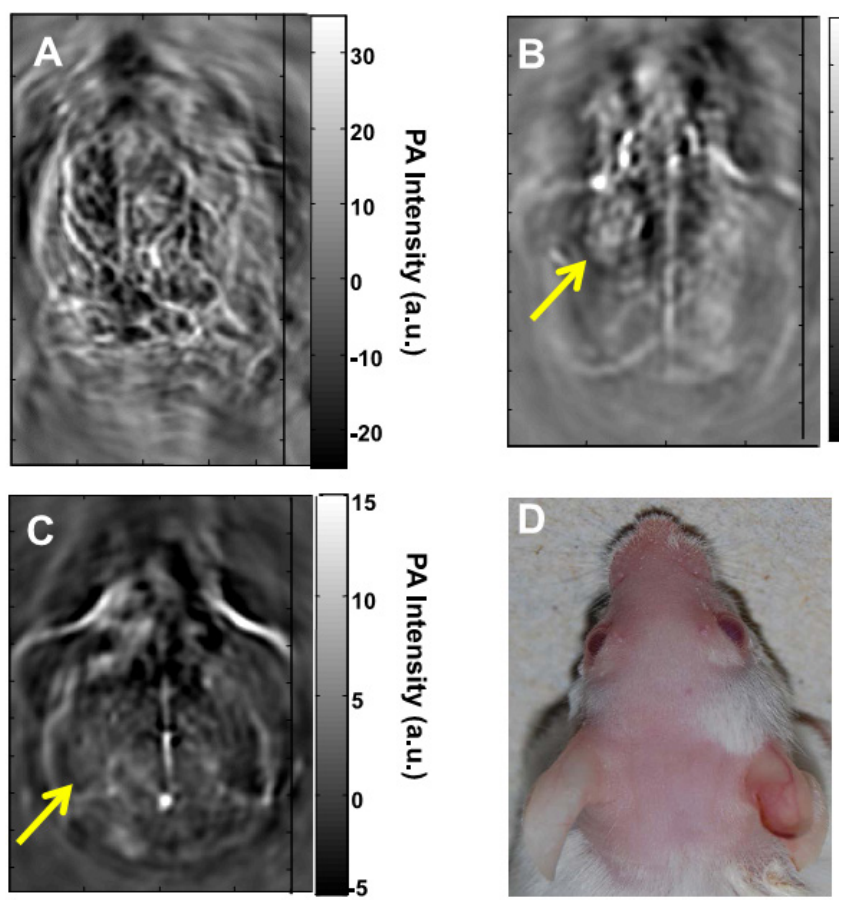

Figure 3. Photoacoustic tomography (PAT) imaging of CuS NPs in mice. (A) A representative PAT image of mouse brain, acquired using a $532 \mathrm{~nm}$ laser without injecting contrast agent. (B) A PAT image of mouse brain acquired using a $1064 \mathrm{~nm}$ laser, at $24 \mathrm{~h}$ after intracranial injection of CuS NPs. (C) A PAT image of mouse brain acquired using a $1064 \mathrm{~nm}$ laser, at 7 days after intracranial injection of CuS NPs. (D) A photograph of the head of the mouse. Images of (A) to (D) are adapted with permission from [12] (C) (2012) American Chemical Society 


\subsubsection{PET/CT imaging}

PET/CT is a highly sensitive and quantitative imaging modality in clinical oncology for cancer diagnosis, staging, and evaluation of therapeutic responses. This imaging modality is principled by the detection of radioactive isotope decay, during which unstable atoms spontaneously convert to a more stable form with a lower overall energy and release radioactive energy [49]. Recently, the radiolabeling of NPs with PET isotopes has gained increasing interest for evaluating the pharmacokinetics, tissue distribution, and clearance. Various radiolabeled NPs have been reported, including quantum dots, gold NPs, carbon nanomaterials, and polymeric NPs.

Conventionally, radioisotopes are linked to NPs through chelators to form stable complexes and this radiometal-chelator methods have two limitations. One limitation is the potential detachment of radioisotopes from NPs in vivo, which can result in misleading findings since PET imaging detects the radioisotopes (whether they are on the nanoparticles or not) but not the NPs themselves. The other limitation is that the data obtained may not reflect the pharmacological properties of unlabeled NPs due to the influence caused by radiotracers on physicochemical properties of NPs. Alternatively, ${ }^{64} \mathrm{Cu}$-labeled $\mathrm{CuS}$ NPs provide a chelator-free method for PET/CT imaging. ${ }^{64} \mathrm{Cu}$ $\left(\mathrm{T}_{1 / 2}=12.7 \mathrm{~h} ; \beta+, 0.653 \mathrm{MeV}[17.8 \%] ; \beta-, 0.579 \mathrm{MeV}\right.$ [38.4\%]) has decay characteristics that allow for both PET imaging and targeted radiotherapy for cancer [8]. In an interesting report, chelator-free, PEG modified, ${ }^{64} \mathrm{Cu}$-labeled CuS NPs ( $\sim 11 \mathrm{~nm}$ in diameter) were constructed to serve as both a PET tracer and a therapy agent in tumor bearing mice (Figure 4). ${ }^{64} \mathrm{CuCl}_{2}$ was used as the precursor for the synthesis of ${ }^{64} \mathrm{Cu}$-labeled CuS NPs, in which ${ }^{64} \mathrm{Cu}$ is an integral building block of $\mathrm{CuS}$ rather than labeling through a chelator. This design with desirable properties such as ease of synthesis, small size, presents an enhanced stability, higher tumor accumulation and hence better imaging results. The PEG- $\left[{ }^{64} \mathrm{Cu}\right] \mathrm{CuS}$ NPs showed high uptake and retention in U87 human glioblastoma xenografts in mice due to the enhanced permeability and retention effect, which was successfully visualized by non-invasive PET imaging. 

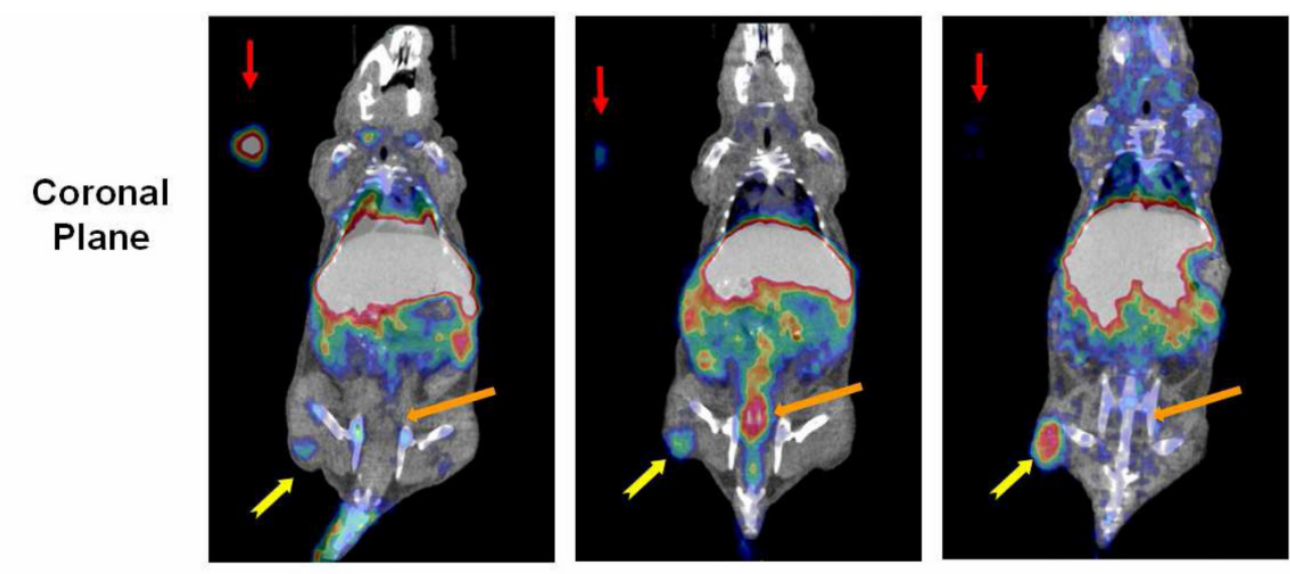

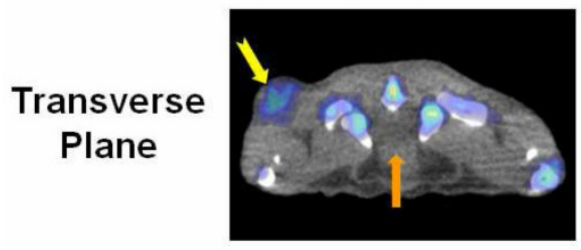

1h

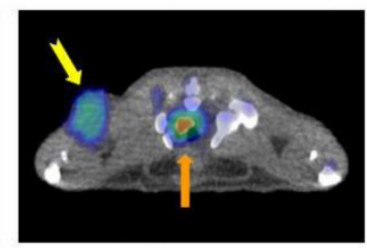

$6 \mathrm{~h}$

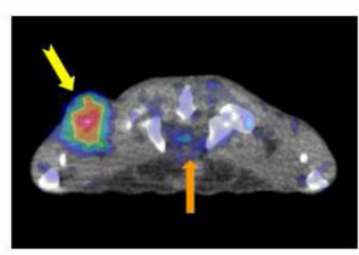

$24 \mathrm{~h}$

Figure 4. Micro-PET/CT images of nude mice-bearing s.c. U87 glioma xenografts acquired at 1,6 , and $24 \mathrm{~h}$ after i.v. injection of PEG- $\left[{ }^{64} \mathrm{Cu}\right]-\mathrm{CuS}$ NPs. Yellow arrow: tumor; orange arrow: bladder; red arrow: standard. These images are adapted with permission from [8] (C) (2010) American Chemical Society

\subsubsection{Photothermal ablation}

Photothermal ablation represents a powerful technique for a variety of applications. This technique usually relies on a NIR laser to irradiate photothermal agents, which then convert the optical energy to heat energy and lead to thermal ablation of surrounded cells or tissues [50]. Many inorganic nanomaterials, such as gold nanostructures, CuS NPs, carbon nanomaterials, copper chalcogenide semiconductors, $\mathrm{Pd}$ nanosheets, $\mathrm{Bi}_{2} \mathrm{Se}_{3}$ nanoplates and $\mathrm{W}_{18} \mathrm{O}_{49}$ nanowires, are widely explored as photothermal agents. Among them, gold nanostructures and CuS NPs represent two main streams.

CuS NPs are a new class of photothermal agents that provide an alternative to gold nanostructures. The NIR absorption of CuS NPs are derived from $\mathrm{d}-\mathrm{d}$ energy band transition of $\mathrm{Cu}^{2+}$ ions [51], and thus their absorption wavelength is not affected by their shape and surrounding environment. In addition, CuS NPs are degradable and can be excreted from liver and kidneys, and thus are favorable for clinical applications. Recently, extensive research has been explored to use the photothermal ablation property of CuS NPs for medical applications, such as photothermal therapy, and transdermal delivery. The detailed summary is listed below [52]. 


\subsubsection{Photothermal therapy}

Several CuS nanostructures are reported as excellent transducer agents for photothermal therapy, including the CuS flower-like superstructures [53] with the mean size of $\sim 1 \mu \mathrm{m}$ and CuS plate-like nanocrystals [10] with a mean size of $\sim 70 \mathrm{~nm} \times 13 \mathrm{~nm}$ (Figure 5). These CuS NPs exhibit strong absorbance and conversion efficiency upon $980 \mathrm{~nm}$ laser irradiation, leading to a higher local temperature to kill the cancer cells.
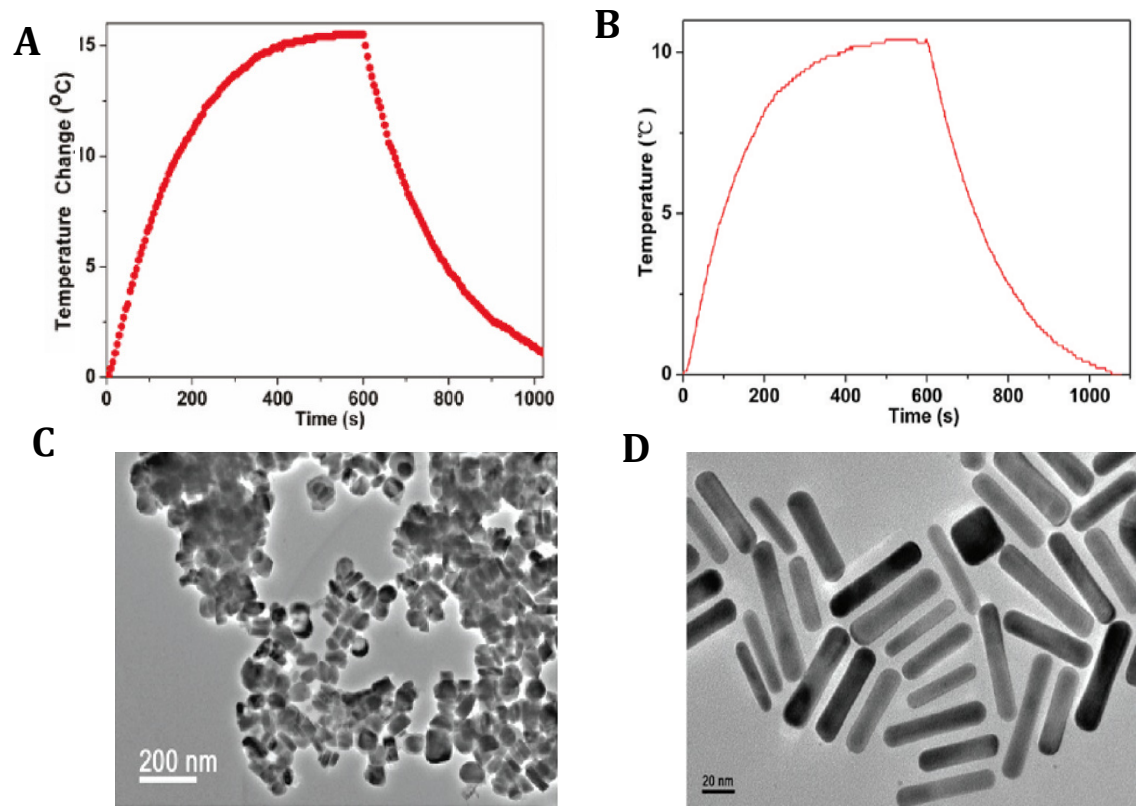

Figure 5. (A) Photothermal effect of the aqueous dispersion of $\mathrm{Cu}_{9} \mathrm{~S}_{5} \mathrm{NCs}(40 \mathrm{ppm})$ with the NIR ( $980 \mathrm{~nm}, 0.51 \mathrm{~W} \mathrm{~cm}^{-2}$ ) irradiation for $10 \mathrm{~min}$. (B) Photothermal effect of

the aqueous dispersion of the Au nanorods (40 ppm) irradiated with 980 laser (a power density of $0.51 \mathrm{~W} \mathrm{~cm}^{-2}$ ) for $10 \mathrm{~min}$. (C) TEM image of the $\mathrm{Cu}_{9} \mathrm{~S}_{5} \mathrm{NCs}$. (D) TEM image of the Au nanorods. Images of (A) to (D) are adapted with permission from [10] (C) (2011) American Chemical Society

Some hollow CuS NPs with numerous mesoporous pores could efficiently load drug molecules, thus providing a combination of photothermal and chemotherapy. For example, hollow CuS NPs with $130 \mathrm{~nm}$ in diameter were loaded with hydrophobic drugs (Camptothecin). Upon NIR irradiation, drugs were demonstrated to be released from the NP systems for chemical cytotoxicity, and the converted heat by CuS NPs offers thermal killing of cells [54]. CuS NP-based photothermal therapy could also be combined with immunotherapy. In an interesting design [11], chitosan-coated hollow CuS NPs were assembled with immunoadjuvants (CpG motifs) to construct a 
nanocomplex. Upon NIR irradiation, photothermal ablation-induced tumor cell death released tumor antigens into the surrounding milieu, and the nanocomplex was re-assembled to form chitosan-CpG complex; which altogether elite and enhance the immune response. The results show that the combinational photothermal-immunotherapy could treat both primary tumor and distant metastasis tumor (Figure 6).

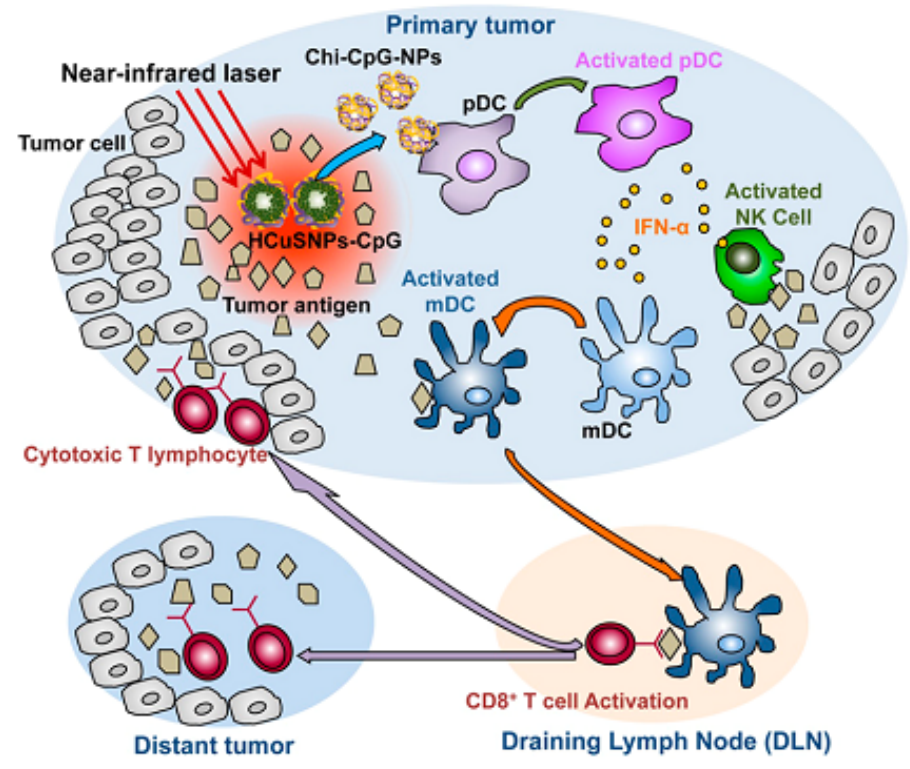

Figure 6. Diagram of HCuSNPs-CpG-mediated photothermal immunotherapy for the treatments of primary and distant tumors. This figure is adapted with permission from [11] (C) (2014) American Chemical Society

\subsubsection{Transdermal delivery}

Compared with other drug delivery routes, transdermal drug delivery provides several advantages, such as sustained and steady-state pharmacokinetics, avoidance of hepatic first-pass effects, and improvement of patient compliance [55]. Taken the advantage of photothermal ablation properties, CuS NPs could be covered on the skin surface to thermally deconstruct the stratum corneum, thus assisting transdermal delivery of drugs. In an interesting report, Ramadan et al. coated the skin surface with gel formations of drug-bearing CuS NPs. They then applied nanosecond-pulsed NIR laser $\left(1.3-2.6 \mathrm{~W} \mathrm{~cm}^{-2}\right)$ to irradiate the skin. The pulsed irradiation could rapidly elevate the local temperature, resulting in the disorder of keratin networks and decompose of stratum corneum, thus facilitating the uptake of drug-bearing CuS NPs. Upon the end of 
pulsed irradiation, the temperature was rapidly cooled down, and thus this method ensures the skin temperature will not exceeds $40-50{ }^{\circ} \mathrm{C}$ in the localized regions and will not produce any severe damage.

\subsubsection{Theranostics}

Theranostics, the integration of therapy and diagnostics in one delivery vehicle, is paving the way towards the goal of personalized medicine. Theranostic nanoconstructs allow for early recognition of diseased sites followed by triggered guidance of therapy. In addition, these theranostic agents employ imaging modalities to monitor the response to disease treatment. Many nanomaterials are explored for theranostic applications, such as iron oxide NPs, quantum dots, carbon nanotubes, gold NPs and silica NPs. Recent years, CuS NPs are also investigated for cancer theranostics because of their many intriguing properties discussed above.

In an interesting report, Zhang et al. designed Cy5.5-conjugated, CuS-loaded, hyaluronic acid (HA) NPs (Figure 7). In this system, Cy5.5 fluorescent signal is quenched by CuS inside the NPs, and HA is fabricated to target cluster determinant 44 (CD44) overexpressed on cancer cells and lymphatic vessel endothelial hyaluronan receptor-1 (LYVE-1). Once HA NPs are delivered into the tumor-bearing mice, they are accumulated in tumor effectively through the enhanced permeation and retention (EPR) effect and then specifically internalized into tumor cells through receptor-mediated endocytosis. Inside the tumor cells, HA NPs will be degraded into short saccharide units by hyaluronidases (mainly Hyal-1 and Hyal-2) and released CuS and Cy5.5. Due to the complete degradation of HA NPs, Cy5.5 will be separately from the quenching of $\mathrm{CuS}$ particles, yielding strong fluorescent signal for tumor imaging; meanwhile, released CuS particles serve as photoacoustic imaging agents to delineate the blood vessels and photothermal agents to destroy the tumor cells. Using this theranostic system, the tumor area could be clearly observed by fluorescence and photoacoustic signals over time, which peaked at the $6 \mathrm{~h}$ time point (tumor to normal tissue ratio of $3.25 \pm 0.25$ for fluorescence imaging and $3.8 \pm 0.42$ for photoacoustic imaging), and the tumor growth could be efficiently inhibited by phothermal ablation, with a tumor inhibition rate of $89.74 \%$ on day 5 . 
A

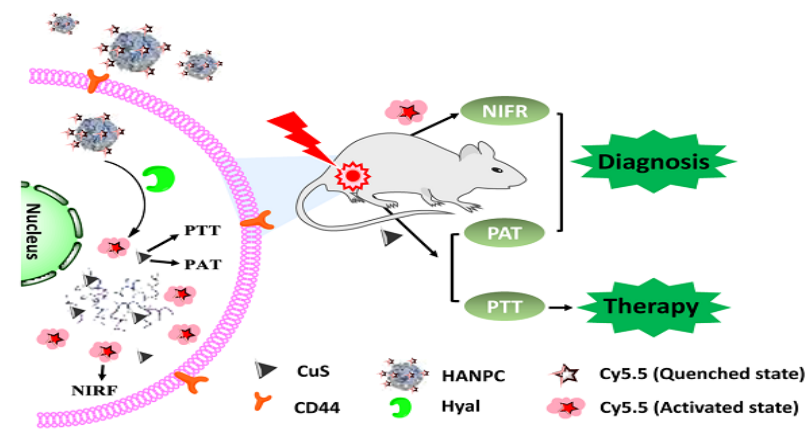

B

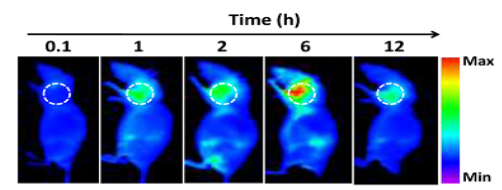

C

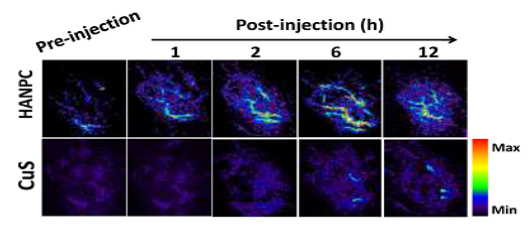

D

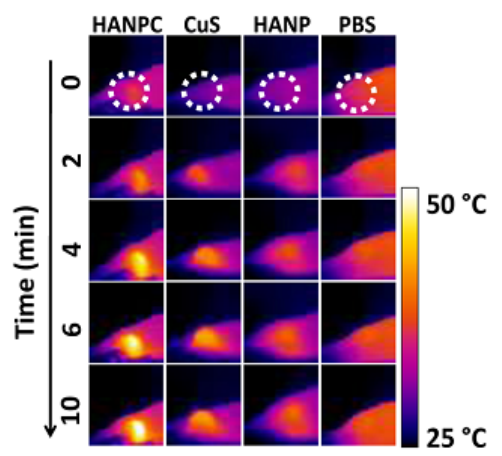

Figure 7. (A) In vivo applications of HANPC for NIR fluorescence and PA image-guided photothermal therapy. (B) NIR fluorescent imaging of CD44-positive SCC7 tumorbearing mice received HANPC i.v. treatment. Images were acquired at indicated time points, and fluorescent signals were normalized by the maximum average value. The color bar indicates radiant efficiency (low, 0 ; high, $0.209 \times 10^{6}$ ). White circles were used to indicate tumors location. (C) Photoacoustic tomography imaging of blood vessels in

SCC7 tumor-bearing mice intravenously received HANPC or free CuS. (D) Thermal images of SCC7 tumor-bearing mice i.v. treated with PBS, HANP, CuS, and HANPC (left row, $5 \mathrm{mg} \mathrm{kg}^{-1}$ of HANPC, illuminated at $6 \mathrm{~h}$ pi.) with $808 \mathrm{~nm}$ laser illumination taken

at indicated time points. The injected amount of HANP and CuS were calculated according to the loading efficiency. The laser power density was $1.5 \mathrm{~W} \mathrm{~cm}^{-2}$. Images of (A) to (D) are adapted with permission from [56] (c) (2014) American Chemical Society

\subsection{SUMMARY}

In this review, we introduce current strategies to synthesize CuS NPs with various morphologies, and their investigations in biomedical applications, including biosensors, molecular imaging, photothermal ablation and theranostics. As a new class of nanomaterials, CuS NPs have the advantages of consistent NIR absorption, low cost, low cytotoxicity and biodegradability, indicating great potential in this research field.

Notably, the development of CuS NPs for biomedical application is still in its infancy, and many challenges exist before translating current animal-based 
research to the clinic. The photothermal conversion efficiency of CuS NPs needs to be improved, probably by optimizing their shape and size. Continuous efforts are needed to enhance their drug delivery capabilities, presumably by modifying the surface chemistry and morphology, or by designing hollow/porous/coreshell architectures with polymeric coating. The combination of chemotherapy, photothermal therapy and molecular imaging will offer numerous new avenues for the design of multifunctional delivery systems. Research on CuS NPs will continue to thrive over the next decade, and tremendous opportunities lie ahead for their potential biomedical applications.

\section{ACKNOWLEDGEMENTS}

This work was supported by National Natural Science Foundation of China (81471779), Thousand Young Talents Program, and the program for professor of special appointment (Eastern Scholar) at Shanghai institutions of higher learning. 


\section{REFERENCES}

1. K. Riehemann, S.W. Schneider, T.A. Luger, B. Godin, M. Ferrari, H. Fuchs. Angew. Chem. Int. Ed. Engl. 48 (2009) 872-897.

2. $\quad$ S. Goel, F. Chen, W. Cai. Small 10 (2014) 631-645.

3. Y. Wu, C. Wadia, W. Ma, B. Sadtler, A.P. Alivisatos. Nano Lett. 8 (2008) 2551-2555.

4. X. Bo, J. Bai, L. Wang, L. Guo. Talanta 81 (2010) 339-345.

5. G. Ku, M. Zhou, S. Song, Q. Huang, J. Hazle, C. Li. ACS Nano 6 (2012) 7489-7496.

6. Z. Xiao. Nanomed. 9 (2014) 373-375.

7. S. Ramadan, L. Guo, Y. Li, B. Yan, W. Lu. Small 8 (2012) 3143-3150.

8. M. Zhou, R. Zhang, M. Huang, W. Lu, S. Song, M.P. Melancon, M. Tian, D. Liang, C. Li. J. Am. Chem. Soc. 132 (2010) 15351-15358.

9. J. Kundu, D. Pradhan. ACS Appl. Mater. Inter. 6 (2014) 1823-1834.

10. Q. Tian, F. Jiang, R. Zou, Q. Liu, Z. Chen, M. Zhu, S. Yang, J.L. Wang, J.H. Wang, J. Hu. ACS Nano 5 (2011) 9761-9771.

11. L. Guo, D.D. Yan, D. Yang, Y. Li, X. Wang, O. Zalewski, B. Yan, W. Lu. ACS Nano 8 (2014) 5670-5681.

12. G. Ku, M. Zhou, S.L. Song, Q. Huang, J. Hazle, C. Li. Acs Nano 6 (2012) 7489-7496.

13. T. Adschiri, K. Kanazawa, K. Arai. J. Am. Ceram. Soc. 75 (1992) 1019-1022.

14. P.W. Dunne, C.L. Starkey, M. Gimeno-Fabra, E.H. Lester. Nanoscale 6 (2014) 2406-2418.

15. X. Liu, G. Xi, Y. Liu, S. Xiong, L. Chai, Y. Qian. J. Nanosci. Nanotechnol. 7 (2007) 4501-4507.

16. J. Zhang, Z. Zhang. Mater. Lett. 62 (2008) 2279-2281.

17. C.O. Kappe, D. Dallinger. Mol. Divers. 13 (2009) 71-193.

18. M.D. Taylor, A.D. Roberts, R.J. Nickles. Nucl. Med. Biol. 23 (1996) 605-609.

19. X.H. Liao, N.Y. Chen, S. Xu, S.B. Yang, J.J. Zhu. J. Cryst. Growth 252 (2003) 593-598.

20. T. Thongtem, A. Phuruangrat, S. Thongtem. Curr. Appl. Phys. 9 (2009) 195-200.

21. W. Wenzhong, X. Haolan, Z. Wei. Mater. Lett. 60 (2006) 2203-2206.

22. M. Kristl, N. Hojnik, S. Gyergyek, M. Drofenik. Mater. Res. Bull. 48 (2013) 1184-1188.

23. X. Yang, W. Lu, J. Hou, X. Li, S. Han. J. Nanosci. Nanotechnol. 11 (2011) 9818-9822.

24. T.H. Larsen, M. Sigman, A. Ghezelbash, R.C. Doty, B.A. Korgel. J. Am. Chem. Soc. 125 (2003) 5638-5639.

25. Y. Li, J. Hu, G. Liu, G. Zhang, H. Zou, J. Shi. Carbohydr. Polym. 92 (2013) 555-563.

26. J. Chen, B.L. Trout. J. Phys. Chem. B 114 (2010) 13764-13772.

27. Y.Y. Kim, D. Walsh. Nanoscale 2 (2010) 240-247.

28. L. Armelao, D. Camozzo, S. Gross, E. Tondello. J. Nanosci. Nanotechnol. 6 (2006) 401-408.

29. M. Orphanou, E. Leontidis, T. Kyprianidou-Leodidou, P. Koutsoukos, K.C. Kyriacou. Langmuir 20 (2004) 5605-5612.

30. M. De, P.S. Ghosh, V.M. Rotello. Adv. Mater. 20 (2008) 4225-4241.

31. G. Yang, C. JingHua, C. Guonan. Sensor. Actuat. B-Chem. 184 (2013) 113-117. 
32. W. Yao, L. Wang, H. Wang, X. Zhang, L. Li, N. Zhang, L. Pan, N. Xing. Biosens. Bioelectron. 40 (2013) 356-361.

33. J. Zou, J. Jiang, L. Huang, H. Jiang, K. Huang, Solid State Sci. 13 (2011) 1261-1267.

34. C.X. Xu, Q.G. Zhai, Y.J. Liu, K.J. Huang, L. Lu, K.X. Li. Anal. Bioanal. Chem. 406 (2014) 6943-6951.

35. C. Ding, Z. Wang, H. Zhong, S. Zhang. Biosens. Bioelectron. 25 (2010) 1082-1087.

36. J.N. Hirschhorn, M.J. Daly. Nat. Rev. Genet. 6 (2005) 95-108.

37. C.C. Mayorga-Martinez, M. Guix, R.E. Madrid, A. Merkoci. Chem. Commun. 48 (2012) 1686-1688.

38. L. Qian, J. Mao, X. Tian, H. Yuan, D. Xiao. Sensor. Actuat. B Chem. 176 (2013) 952-959.

39. A. Louie. Chem. Rev. 110 (2010) 3146-3195.

40. S.-H. Seo, B.-M. Kim, A. Joe, H.-W. Han, X. Chen, Z. Cheng, E.S. Jang. Biomaterials 35 (2014) 3309-3318.

41. M. Ferrari. Nat. Rev. Cancer 5 (2005) 161-171.

42. C.R. Patra, R. Bhattacharya, D. Mukhopadhyay, P. Mukherjee. Adv. Drug Deliv. Rev. 62 (2010) 346-361.

43. X. Huang, S. Lee, X. Chen. Am. J. Nucl. Med. Mol. Imaging 1 (2011) 3-17.

44. C.C. Kuo, P. Luu, K.K. Morgan, M. Dow, C. Davey. Plos One 9 (2014) e112103.

45. J. Yan, J.C. Lim, D.W. Townsend. Phys. Med. Biol. 60 (2015) 961-976.

46. P. Padilla, J.M. Gorriz, J. Ramirez, D. Salas-Gonzalez, I.A. Illan. Neurocomputing 150 (2015) 4-15.

47. J. Jose, S. Manohar, R.G.M. Kolkman, W. Steenbergen, T.G. van Leeuwen. J. Biophotonics 2 (2009) 701-717.

48. K. Yang, L. Zhu, L. Nie, X. Sun, L. Cheng, C. Wu, G. Niu, X. Chen, Z. Liu. Theranostics 4 (2014) 134-141.

49. S. Chiang. Oral Maxillofac. Surg. Clin. North Am. 26 (2014) 239-245.

50. M. Zaverl, O. Valerio, M. Misra, A. Mohanty. J. Appl. Polym. Sci. 132 (2015) 41278-41288.

51. Y. Li, W. Lu, Q. Huang, M. Huang, C. Li, W. Chen. Nanomed.-Nanotechnol. 5 (2010) 1161-1171.

52. L. Guo, I. Panderi, D.D. Yan, K. Szulak, Y. Li, Y.-T. Chen, H. Ma, D.B. Niesen, N. Seeram, A. Ahmed, B. Yan, D. Pantazatos, W. Lu. Acs Nano 7 (2013) 8780-8793.

53. Q. Tian, M. Tang, Y. Sun, R. Zou, Z. Chen, M. Zhu, S. Yang, J. Wang, J. Wang, J. Hu. Adv. Mater. 23 (2011) 3542-3547.

54. $\quad$ K. Dong, Z. Liu, Z. Li, J. Ren, X. Qu. Adv. Mater. 25 (2013) 4452-4458.

55. S. Ramadan, L. Guo, Y. Li, B. Yan, W. Lu. Small 8 (2012) 3143-3150.

56. L. Zhang, S. Gao, F. Zhang, K. Yang, Q. Ma, L. Zhu. Acs Nano 8 (2014) 12250-12258. 